\title{
MODEL COMPLETENESS OF AN ALGEBRA OF LANGUAGES ${ }^{1}$
}

\author{
DAVID HAUSSLER
}

\begin{abstract}
An algebra $\langle\mathcal{L}, f, g\rangle$ of languages over a finite alphabet $\Sigma=$ $\left\{a_{1}, \ldots, a_{n}\right\}$ is defined with operations $f\left(L_{1}, \ldots, L_{n}\right)=a_{1} L_{1} \cup \cdots \cup a_{n} L_{n} \cup$ $\{\lambda\}$ and $g\left(L_{1}, \ldots, L_{n}\right)=a_{1} L_{1} \cup \cdots \cup a_{n} L_{n}$ and its first order theory is shown to be model complete. A characterization of the regular languages as unique solutions of sets of equations in $\langle\mathcal{L}, f, g\rangle$ is given and it is shown that the subalgebra $\langle\mathscr{R}, f, g\rangle$ where $\mathscr{R}$ is the set of regular languages is a prime model for the theory of $\langle\mathcal{L}, f, g\rangle$. We show also that the theory of $\langle\mathcal{E}, f, g\rangle$ is decidable.
\end{abstract}

Let $\Sigma=\left\{a_{1}, \ldots, a_{n}\right\}$ be a finite alphabet and $\Sigma^{*}$ the free semigroup with empty word $\lambda$ generated by $\Sigma$. Let $\mathcal{L}$ be the class of all languages over $\Sigma$, i.e., all subsets of $\Sigma^{*}$. We introduce two $n$-ary operations on the languages of $\mathcal{L}$ :

$$
\begin{aligned}
& f\left(L_{1}, \ldots, L_{n}\right)=a_{1} L_{1} \cup \cdots \cup a_{n} L_{n} \cup\{\lambda\}, \\
& g\left(L_{1}, \ldots, L_{n}\right)=a_{1} L_{1} \cup \cdots \cup a_{n} L_{n},
\end{aligned}
$$

where $a_{i} L_{i}$ denotes the language obtained by prefixing all the words of $L_{i}$ with the letter $a_{i}$.

Our first result is the following theorem which follows from Theorem 3 of Mycielski and Perlmutter [3].

THEOREM 1. The first order theory of the algebra $\langle\mathcal{L}, f, g\rangle$ is model complete.

Proof. Let us define a simple bijection between $\mathcal{L}$ and the set of infinite, oriented trees with nodes labeled from $\{f, g\}$, each node having $n$ successors. Given such a tree, label the edges emanating from each node with the letters $a_{1}$ through $a_{n}$ from left to right. Associate with the tree the language consisting of all words of $\Sigma^{*}$ corresponding to the consecutive labels of the edges of any path leading from the root to a node labeled $f$. It follows that the algebra $\langle\mathcal{L}, f, g\rangle$ is isomorphic to the algebra $R_{\sigma}$ of [3], where $\sigma$ specifies the two $n$-ary function

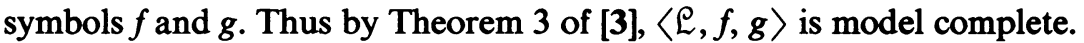

We now consider sets of equations for $\langle\mathcal{L}, f, g\rangle$, i.e., sets of equations written solely in terms of the function symbols $f$ and $g$ and variables $x_{i}$.

Let us say that a language $L$ is uniquely determined by a set of equations $E$ and a variable $x_{p}$ iff $E$ is satisfiable in $\langle\mathcal{E}, f, g\rangle$ and every assignment to the variables of $E$ which satisfies $E$ assigns $L$ to $x_{p}$.

Received by the editors November 3, 1980.

1980 Mathematics Subject Classification. Primary 03C60, 68D30.

'This research was conducted while the author was supported by a University of Colorado doctoral fellowship. 
LEMMA 1. If $L$ is uniquely determined by some set of equations and a variable, then $L$ is uniquely determined by $a$ set of equations $E$ and $x_{1}$, where $E$ has the unknowns $x_{1}, \ldots, x_{m}$ and is of the form $\left\{x_{i}=t_{i}: 1<i<m\right\}$, the $t_{i}$ 's being terms which are not variables.

Proof. Assume that $L$ is uniquely determined by the set of equations $D$ and the variable $x_{p}$. We define an equivalence relation, $\equiv$, on the variables appearing in $D$ by

$$
x_{i} \equiv x_{j} \quad \text { iff } D \Rightarrow x_{i}=x_{j} \text { is true in }\langle\mathcal{E}, f, g\rangle .
$$

From each equivalence class, we choose a representative, insuring that $x_{p}$ is chosen as a representative of its class. We then replace all the variables in $D$ by their representatives, obtaining a set of equations $D^{\prime}$ which is equivalent to $D$ with respect to the remaining variables.

Using the isomorphism from Theorem 1 and Lemma B, Case I from [3], we convert $D^{\prime}$ to an equivalent set of equations $D^{\prime \prime}=\left\{x_{i_{k}}=t_{k}: 1<k<r\right\}$ where the $x_{i_{k}}$ 's are distinct variables and the $t_{k}$ 's are terms which are not variables. Now notice that the system $D^{\prime \prime}$ is satisfiable in $\langle\mathcal{L}, f, g\rangle$ for every assignment of the variables which do not occur on the left-hand side of any equation of $D^{\prime \prime}$ (see [3, Formula (2)]). Hence $x_{p}$ appears on the left-hand side of some equation in $D^{\prime \prime}$. To finish the proof, we substitute every variable of $D^{\prime \prime}$ which does not appear on the left-hand side of any equation by the variable $x_{p}$. Finally, we rename the variables to obtain a set of equations $E$ of the desired form.

Our second theorem provides a characterization of the class of regular languages (see e.g. [2]) in terms of sets of equations in $\langle\mathfrak{L}, f, g\rangle$.

THEOREM 2. The following are equivalent.

(i) $L$ is uniquely determined by some set of equations and a variable in $\langle\mathcal{L}, f, g\rangle$.

(ii) $L$ is uniquely determined by $a$ set of equations $E$ in unknowns $x_{1}, \ldots, x_{m}$ and the variable $x_{1}$, where $E$ is of the form $\left\{x_{i}=\phi_{i}\left(x_{i_{1}}, \ldots, x_{i_{n}}\right): 1<i<m\right\}$ and $\phi_{i} \in\{f, g\}$ for each $i$.

(iii) $L$ is regular.

Proof. We first show (i) $\Rightarrow$ (ii). By Lemma 1 , we may assume that $L$ is uniquely determined by $E_{0}=\left\{x_{i}=t_{i}: 1 \leqslant i \leqslant m\right\}$ and the variable $x_{1}$ where $E_{0}$ has the properties stated in the lemma. From $E_{0}$ we will produce a set of equations $E$ of the form specified in (ii) in the following way. Initially let $E=E_{0}$. Then, given any equation of $E$ of the form $x_{j}=\phi\left(u_{1}, \ldots, u_{n}\right)$ where the $u_{i}$ 's are terms and for some $k: 1 \leqslant k \leqslant n, u_{k}$ is not a variable, replace this equation with the two equations $x_{j}=\phi\left(u_{1}, \ldots, u_{k-1}, x_{i}, u_{k+1}, \ldots, u_{n}\right)$ and $x_{i}=u_{k}$ where $i$ is the least integer such that $x_{i}$ does not appear in any equations of $E$ up to this point. We continue this operation as long as feasible. Since terms are of finite depth, this process terminates and it is apparent that it produces a set of equations $E$ of the required form which is equivalent to $E$ with respect to the original variables.

To show (ii) $\Rightarrow$ (iii), we transform $E$ into a finite automaton $M=$ $\left\langle Q, \Sigma, \delta, x_{1}, F\right\rangle$ accepting precisely the language $L . Q$, the set of states of $M$, is 
defined to be the set of variables of $E . \Sigma=\left\{a_{1}, \ldots, a_{n}\right\}$ is the alphabet of $M . \delta$, the transition function, is defined by $\delta\left(x_{k}, a_{j}\right)=x_{i}$ iff $E$ has an equation of the form $x_{k}=\phi\left(x_{i_{1}}, \ldots, x_{i_{n}}\right) . x_{1}$ is the start state and $F$, the set of accepting states, is the set of those variables $x_{k}$ for which an equation of the form $x_{k}=f\left(x_{i_{1}}, \ldots, x_{i_{n}}\right)$ is in $E$. In view of the definition of the operations $f$ and $g$, it is obvious that $M$ must accept $L$, hence $L$ is regular.

To see that (iii) $\Rightarrow$ (i) it suffices to observe that given any deterministic finite automaton $M=\left\langle Q, \Sigma, \delta, x_{1}, F\right\rangle$ with $Q \subset\left\{x_{1}, x_{2}, \ldots\right\}$ and $\Sigma=\left\{a_{1}, \ldots, a_{n}\right\}$ we can easily reverse the above construction, obtaining a set of equations $E$ such that $E$ and $x_{1}$ uniquely determine the language $L$ accepted by $M$.

Let $R$ be the class of regular languages over $E$. Since $R$ is closed under the operations $f$ and $g,\langle\Re, f, g\rangle$ is a subalgebra of $\langle\mathcal{L}, f, g\rangle$. From Theorem 2 we may easily deduce the following corollary.

COROllary 1. Every finite set of equations in $f$ and $g$ which has a solution in $\langle\mathcal{L}, f, g\rangle$ has a solution in $\langle\Re, f, g\rangle$.

However, using [3] again, we obtain the following stronger result.

THEOREM 3. $\langle\mathcal{R}, f, g\rangle$ is an elementary subalgebra of. $\langle\mathcal{E}, f, g\rangle$ and is a prime model for its theory.

Proof. By Theorem 2 and the isomorphism of the proof of Theorem $1,\langle\Re, f, g\rangle$ is isomorphic to the algebra $A_{\sigma}$ of [3] where $\sigma$ is as before. Our result follows from part (ii) of Theorem 3 of [3].

THEOREM 4. The theory of $\langle\mathfrak{L}, f, g\rangle$ is decidable.

Proof. We can define the operations $f$ and $g$ in the monadic second-order theory $\langle S n S\rangle$ of $n$ successor functions $S_{1}, \ldots, S_{n}$ as follows.

$$
\begin{aligned}
& g\left(L_{1}, \ldots, L_{n}\right)=L \underset{\text { def }}{\Leftrightarrow} \forall x\left[x \in L \Leftrightarrow \underset{1<i<n}{\bigvee} \exists y \in L_{i}\left[x=S_{i}(y)\right]\right] \\
& f\left(L_{1}, \ldots, L_{n}\right)=L \underset{\text { def }}{\Leftrightarrow} \forall x\left[x \in L \Leftrightarrow\left(\bigvee_{1<i<n} \exists y \in L_{i}\left[x=S_{i}(y)\right]\right) \vee x=\lambda\right],
\end{aligned}
$$

where

$$
x=\lambda \underset{\text { def }}{\forall y} \bigwedge_{1<i<n} x \neq S_{i}(y)
$$

Since Rabin [4] has shown that $\langle S n S\rangle$ is decidable, our theorem follows.

ReMarK. By a slight modification of the proof, Theorem 4 generalizes to the algebra $R_{\sigma}$ of [3].

Problem. In [1], J. H. Conway defines and studies the operations:

$$
\partial(L) / \partial a_{i}=\left\{w: a_{i} w \in L\right\}
$$

which are existentially first order definable from $f$ and $g$. Does the theory of the algebra $\left\langle\mathcal{E}, f, g, \partial / \partial a_{1}, \ldots, \partial / \partial a_{n}\right\rangle$ admit elimination of quantifiers?

ACKNOWLEDGEMENT. I am indebted to Jan Mycielski for numerous refinements of my original observations and proofs. 


\section{REFERENCES}

1. J. H. Conway, Regular algebra and finite machines, Chapman \& Hall, London, 1971.

2. J. E. Hopcroft and J. D. Ullman, Introduction to automata theory, languages and computation, Addison-Wesley, Reading, Mass., 1979.

3. J. Mycielski and P. Perlmutter, Model completeness of some metric completions of absolutely free algebras, Algebra Universalis 12 (1981) (to appear).

4. M. O. Rabin, Decidability of second-order theories and automata on infinite trees, Trans. Amer. Math. Soc. 141 (1969), 1-35.

Department of Computer Science, University of Colorado at Boulder, Boulder, Colorado 80309 